\title{
Alignment of microbial fitness with engineered product formation: obligatory coupling between acetate production and photoautotrophic growth
}

Wei Du', Joeri A. Jongbloets ${ }^{1}$, Coco van Boxtel ${ }^{2}$, Hugo Pineda Hernández ${ }^{1}$, David Lips ${ }^{1}$, Brett G. Oliver ${ }^{2,3}$, Klaas J. Hellingwerf ${ }^{1}$ and Filipe Branco dos Santos ${ }^{*}$ (D)

\begin{abstract}
Background: Microbial bioengineering has the potential to become a key contributor to the future development of human society by providing sustainable, novel, and cost-effective production pipelines. However, the sustained productivity of genetically engineered strains is often a challenge, as spontaneous non-producing mutants tend to grow faster and take over the population. Novel strategies to prevent this issue of strain instability are urgently needed.

Results: In this study, we propose a novel strategy applicable to all microbial production systems for which a genome-scale metabolic model is available that aligns the production of native metabolites to the formation of biomass. Based on well-established constraint-based analysis techniques such as OptKnock and FVA, we developed an in silico pipeline-FRUITS - that specifically'Finds Reactions Usable in Tapping Side-products'. It analyses a metabolic network to identify compounds produced in anabolism that are suitable to be coupled to growth by deletion of their re-utilization pathway(s), and computes their respective biomass and product formation rates. When applied to Synechocystis sp. PCC6803, a model cyanobacterium explored for sustainable bioproduction, a total of nine target metabolites were identified. We tested our approach for one of these compounds, acetate, which is used in a wide range of industrial applications. The model-guided engineered strain shows an obligatory coupling between acetate production and photoautotrophic growth as predicted. Furthermore, the stability of acetate productivity in this strain was confirmed by performing prolonged turbidostat cultivations.
\end{abstract}

Conclusions: This work demonstrates a novel approach to stabilize the production of target compounds in cyanobacteria that culminated in the first report of a photoautotrophic growth-coupled cell factory. The method developed is generic and can easily be extended to any other modeled microbial production system.

Keywords: Strain stability, Growth coupled, Metabolic modeling, Cyanobacteria, Acetate production

\section{Background}

The advent of genetic engineering in the 1970s imbued us with the ability to rationally tailor microorganisms to our needs [1]. Although immediately recognized for its

\footnotetext{
*Correspondence: f.brancodossantos@uva.nl

${ }^{1}$ Molecular Microbial Physiology Group, Faculty of Science, Swammerdam Institute for Life Sciences, University of Amsterdam, Science Park 904, 1098 XH Amsterdam, The Netherlands

Full list of author information is available at the end of the article
}

biotechnological potential [2], four-and-a-half decades later, the usage of genetically engineered organisms in large-scale industrial processes still faces some significant technical hurdles [3]. An important example of such challenges is the process unpredictability that emerges from the instability of engineered strains in production settings [4]. This occurs because conventional metabolic engineering strategies often cause high fitness trade-offs for production strains, as product is made in direct competition with biomass formation (Fig. 1a). This ultimately 


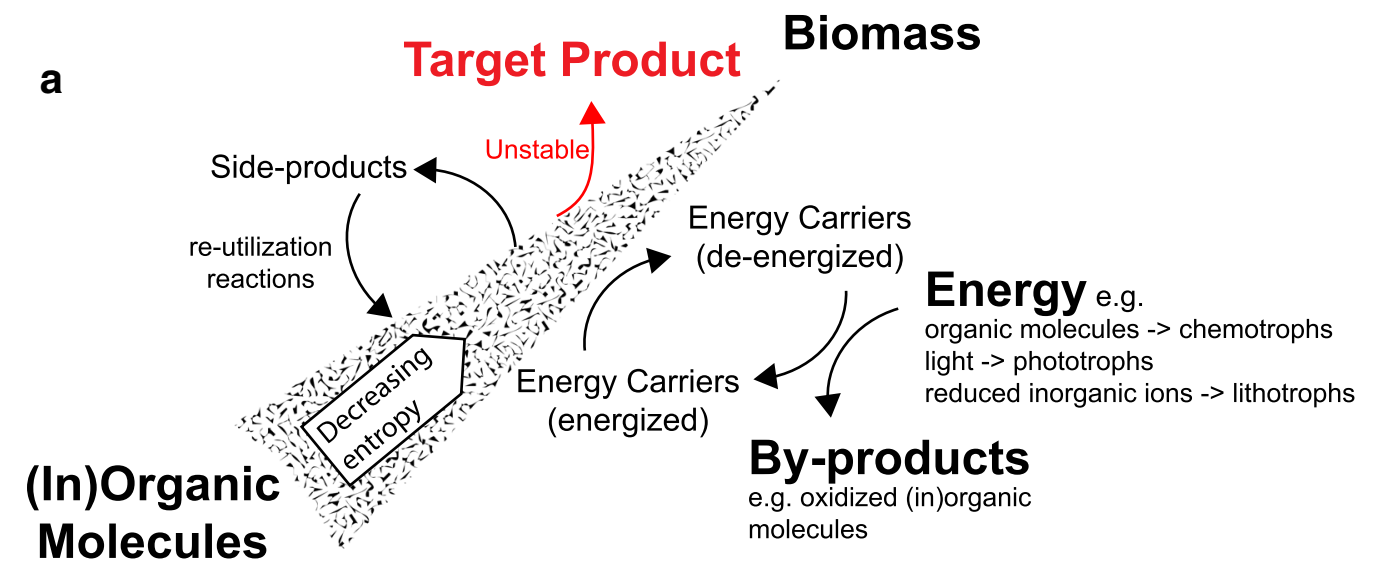

\section{Biomass}

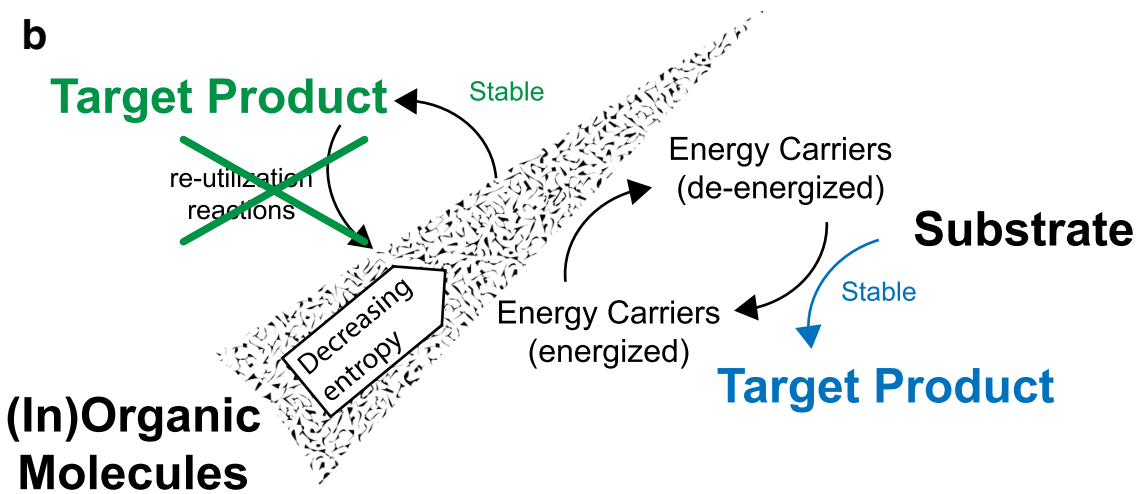

Fig. 1 Schematic representation of the main mass and energy balances involved in biomass formation, along with alternative metabolic engineering strategies to make target compounds. a Target product formation is metabolically engineered using classical approaches that redirect native fluxes toward it. Product formation and biomass formation compete for carbon and energy, which eventually leads to strain instability. $\mathbf{b}$ Overview of growth-coupled metabolic engineering strategies. Others have proposed to tie fluxes to biomass and target product by linking energy and/or redox regeneration to product formation (blue). We propose here to achieve this by knocking out the genes encoding pathways responsible for re-utilizing the side products of anabolism (green)

leads to a rapid appearance of suppressor mutations, for instance in the form of insertions or deletions, which impair the culture's ability to form the product $[5,6]$.

Cyanobacteria that can use (sun) light as their sole energy source can be genetically engineered to directly convert $\mathrm{CO}_{2}$ and water into compounds of interest [7]. This is highly relevant for its potential to mitigate climate change and thus contribute to the increased sustainability of society. However, also here the emergence of suppressor mutations strongly compromises the economic viability of such processes, making them difficult to scaleup without significant productivity losses [8]. In laboratory experiments, engineered strains have been reported to be unstable for the products ethylene [9], lactate [10], isopropanol [11] and mannitol [12]. Given that negative results are often ignored in science, there have likely been many more occurrences of genetic instability in practice, to the point that the genetic instability problem in cyanobacteria has been called an "elephant in the room," as it is "important, obvious, yet largely ignored" [13]. Recently, we have directly demonstrated that the fitness burden of product synthesis is associated with deviating carbon itself from biomass to product formation [14]. This unsettling observation suggests that a certain degree of culture instability is apparently inevitable using the genetic engineering approaches implemented for photoautotrophs thus far.

Enforcing obligate coupling of bacterial growth with the synthesis of specific target products-commonly termed growth-coupled production-can help stabilize production traits. By aligning microbial fitness with product formation, Darwinian selection ensures that spontaneously 
occurring non-producing mutants are outcompeted by the fitter producing strains from the growing population [15]. One can find organisms for which this concept has evolved in nature for a limited range of products. As an example, many fermentative chemoheterotrophs secrete products, such as butanol, acetone and ethanol, in a growth-coupled fashion to maintain a closed redox balance in the cell [16].

Significant efforts have been made, both in silico and in vivo, to engineer growth-coupled production in microbes. For instance, in Escherichia coli there are several successful cases of growth-coupled product formation being reported for lactate [17], 1-butanol [18], succinate and malate [19] and iso-butanol [20]. However, a recent screen based on the analysis of its genome-scale metabolic model (GSM) concluded that many more products can potentially be produced using this approach [21]. Remarkably, the latter authors found that the production of all metabolites present in central metabolism could in principle be obligatorily coupled to growth under aerobic conditions. Although no experimental evidence has been provided for this claim so far, this suggests that stable product formation may be engineered for several compounds.

The idea of growth-coupled production has also been proposed and discussed in the literature for cyanobacteria [22-26], but has never been implemented experimentally. Similar to what has been done for chemoheterotrophs, the theoretical framework applied to cyanobacteria involved coupling a product-forming pathway to the ability of a metabolic network to regenerate energy and/or redox co-factors. This has been achieved in GSM simulations by closing or limiting the in silico flux through any pathway that is responsible for it in the wild-type organism (Fig. 1b), while simultaneously ensuring that the flux through the proposed production pathway is the only feasible alternative to produce energy and/or balance the redox potential. In Synechocystis sp. PCC6803 (hereafter, Synechocystis), a very detailed analysis has been carried out for the production of reduced solvents (e.g., ethanol), finding that at least five genes associated with alternative electron flow need to be blocked to ensure growth coupling [25]. Even with the latest CRISPR-based mutagenesis technology [27, 28], the attempt to test such predictions will still require significant time and effort, without guaranteed in vivo results. More importantly, deletion of alternative electron flow pathways would undoubtedly lead to a strain that is more fragile under varying light conditions [29] and hence be very difficult to cultivate in industrial settings.

Here, we present a strategy to align microbial fitness with product formation that is, in principle, applicable to any microbial production system. Rather than using energy or redox regeneration, we instead propose to directly (i.e., stoichiometrically and obligatorily) couple the production of target compounds to pathways uniquely responsible for the formation of biomass precursors. This can be achieved by deleting the native metabolic routes that cells have to reintroduce side products formed in anabolic pathways. This will lead to their accumulation and ensure growth-coupled production (Fig. 1b).

We have developed a new algorithm to analyze GSMs that allows identification of target products, along with the necessary modifications to the network to guarantee that their formation is growth coupled. We tested this approach on a published metabolic network reconstruction available for Synechocystis, and found in silico that the synthesis of at least nine products could be stoichiometrically coupled to the formation of biomass precursors. This approach was then experimentally validated for one of these predicted products, acetate, to showcase its feasibility. We have thus achieved the first growthcoupled cyanobacterial cell factory, using an approach that can be applied to any organism for which a GSM is available.

\section{Methods}

\section{In silico simulation and tools}

The genome-scale stoichiometric model $i$ JN678 [30] was used to simulate light-limited photoautotrophic growth of Synechocystis. The model topology and its numerical parameters (e.g., reaction stoichiometry, biomass equation composition, and lower and upper bounds of metabolic fluxes) were left unchanged. With these default settings, using the maximization of biomass formation as the objective function, the selected lower bound of the photon uptake flux $\left(30 \mu \mathrm{mol}\right.$ photons $\left.\mathrm{m}^{-2} \mathrm{~s}^{-1}\right)$ limits the biomass synthesis rate $\left(0.052 \mathrm{gDW} \mathrm{h}^{-1}\right)$. The newly developed algorithm was implemented as a single script within a Python package containing the complete analysis pipeline (https://gitlab.com/mmp-uva/fruits). All constraint-based analysis techniques used within this pipeline, namely flux balance analysis (FBA) [31], flux variability analysis (FVA) [32] and OptKnock [33], were performed using PySCeS-CBMPy [34] (http://cbmpy sourceforge.net) in combination with ILOG CPLEX Optimization Studio (IBM) under an academic license. Additional visualization of modeling simulations were carried out using a resource particularly developed for Synechocystis [35] available at the FAME online modeling environment [36].

\section{Strains and general cultivation conditions}

All molecular cloning procedures were carried out in E. coli $\mathrm{DH} 5 \alpha$, either grown on solidified LB plates, 
containing $1.5 \%(\mathrm{w} / \mathrm{v})$ agar, or in liquid LB medium at $37^{\circ} \mathrm{C}$ in an incubator with a shaking speed of $200 \mathrm{rpm}$. When appropriate, antibiotics were added to the medium for propagation of specific plasmids. Concentrations of antibiotics used, alone or in combination, were $100 \mu \mathrm{g} \mathrm{mL}^{-1}$ for ampicillin and $50 \mu \mathrm{g} \mathrm{mL}^{-1}$ for kanamycin.

A glucose-tolerant Synechocystis derivative was obtained from D. Bhaya, University of Stanford, Stanford, CA. Normally, it was cultivated in BG11 medium [37] at $30{ }^{\circ} \mathrm{C}$ in a shaking incubator at $120 \mathrm{rpm}$ (Innova 43 , New Brunswick Scientific) under constant moderate white light illumination, $\sim 30 \mu \mathrm{mol}$ photons $\mathrm{m}^{-2} \mathrm{~s}^{-1}$, measured with an LI-250 light meter (LI-COR, Inc). For Synechocystis mutant construction, kanamycin or nickel sulfate was added to the medium with a final concentration of $50 \mu \mathrm{g} \mathrm{mL} \mathrm{m}^{-1}$ or $20 \mu \mathrm{M}$, respectively. Biomass concentration in the cultures was monitored by recording the optical density at $730 \mathrm{~nm}\left(\mathrm{OD}_{730}\right)$ in a spectrophotometer (Lightwave II, Biochrom).

\section{Plasmid and Synechocystis mutant engineering}

All strains and plasmids are listed in Table 1. To construct the markerless gene knockout strains, a counterselection approach was used [38]. This approach uses two plasmids to markerlessly delete a locus from the Synechocystis chromosome. First one selection plasmid, containing the up- and downstream homologous regions ( $\sim 1 \mathrm{~kb}$ each) of the target locus, and a selection cassette in between, is used to replace the native sequence. Then, another plasmid with only the up- and downstream homologous regions is used to remove the selection cassette. The selection cassette consists of kanamycin resistance and nickel-induced mazF expression fragments [38]. MazF acts as a general inhibitor for the synthesis of all cellular proteins by displaying endoribonuclease activity that cleaves mRNA at the ACA triplet sequence [39]. To construct the required plasmids, first the corresponding sets of the homologous regions of acs and ack $A$ were amplified from the genomic DNA of Synechocystis, and separately fused together using $P f u$ DNA Polymerase (Thermo Scientific). After gel extraction and purification (Zymo Research), an extra adenosine ("A") was attached to the $3^{\prime}$ end of each fused fragment using Taq DNA Polymerase (Thermo Scientific). TA cloning enables ligation of these fragments to the BioBrick " $\mathrm{T}$ " vector pFLSN [40], resulting in pWD001 and pWD003, respectively. To obtain the selection plasmid, either an $\mathrm{Xba \textrm {I }}$ or AvrII restriction site was introduced between the homologous regions through designed fusion primers for, respectively, pWD001 and pWD003. Since the selection cassette (from pWD42) contains an XbaI restriction site on both sides, it could easily be inserted into pWD001 and pWD003, resulting in pWD002 and pWD004, respectively. All the fragments amplified in this study were confirmed by Sanger sequencing at Macrogen Europe (The Netherlands), and all the primers used are listed in Additional file 1: Table S1.

Synechocystis mutant construction takes two rounds of transformation to achieve a markerless gene deletion. The first round is to fully integrate the selection cassette into the chromosome through homologous recombination, while the second round is to completely remove it again. To transform Synechocystis with the plasmids, fresh cells were collected either directly from the plate or from liquid culture $\left(\mathrm{OD}_{730} \approx 1\right)$. After being washed twice with fresh BG11 medium through centrifugation $(5000 \mathrm{rpm}$, $5 \mathrm{~min}$ ), cells were further concentrated to a total volume of $200 \mu \mathrm{L}\left(\mathrm{OD}_{730} \approx 2\right)$. The desired plasmid was mixed with these cells to a concentration of $10 \mu \mathrm{g} \mathrm{mL}{ }^{-1}$, and then the mixture was illuminated with white light of moderate intensity $\left(\sim 50 \mu \mathrm{mol}\right.$ photons $\left.\mathrm{m}^{-2} \mathrm{~s}^{-1}\right)$ for

Table 1 Plasmids and strains used in this study

\begin{tabular}{|c|c|c|}
\hline Plasmids and strains & Relevant characteristics & References \\
\hline $\mathrm{pFL}-\mathrm{SN}$ & BioBrick "T"vector with Spel and Nhel on each side & {$[40]$} \\
\hline pWD42 & Amp ${ }^{r} \mathrm{Km}^{r}$, containing the selection cassette & {$[58]$} \\
\hline pWD001 & pFL-SN derivate, Ampr', containing acs gene upstream and downstream homologous regions & This study \\
\hline pWD002 & $\begin{array}{l}\text { pFL-SN derivate, Amp }{ }^{r} \mathrm{Km}^{r} \text {, containing acs gene upstream homologous region, selection cassette and down- } \\
\text { stream homologous region }\end{array}$ & This study \\
\hline pWD003 & pFL-SN derivate, Ampr', containing ackA gene upstream and downstream homologous regions & This study \\
\hline pWD004 & $\begin{array}{l}\text { pFL-SN derivate, Amp }{ }^{r} \mathrm{Km}^{r} \text {, containing ackA gene upstream homologous region, selection cassette and } \\
\text { downstream homologous region }\end{array}$ & This study \\
\hline Synechocystis sp. PCC6803 & Synechocystis sp. PCC6803 wild type & D. Bhaya \\
\hline WD023 & Synechocystis sp. PCC6803 acs gene knock out mutant & This study \\
\hline WD025 & Synechocystis sp. PCC6803 ackA gene knock out mutant & This study \\
\hline WD027 & Synechocystis sp. PCC6803 acs and ackA double gene knock out mutant & This study \\
\hline
\end{tabular}


4-6 h. Next, the mixture was spread on a commercial membrane (Pall Corporation), resting on a BG11 agar plate (without marker selection). After further illumination for about 16-24 $\mathrm{h}$, the membrane containing the mixture of cells was transferred to a new BG11 plate, supplemented with kanamycin (first round) or nickel sulfate (second round). After about 1 week, colonies were picked and streaked sequentially on a new BG11 plate with kanamycin and a plate with nickel sulfate (first round), or a new BG11 plate with nickel sulfate and a plate with kanamycin (second round). Colonies which grew on the BG11 plate with kanamycin but not on plate with nickel sulfate (first round), or on a BG11 plate with nickel sulfate but not on a plate with kanamycin (second round), were candidates for PCR confirmation. Further segregation by serial dilution in liquid culture under the same conditions was applied when necessary.

\section{Batch cultivation}

Batch cultivations were carried out in the Multi-Cultivator (MC1000-OD, PSI, Czech Republic), with emitted light intensity controlled through a LED panel, equipped with "cool-white" LEDs (PSI, CZ). All experiments were performed at $30^{\circ} \mathrm{C}$ in BG11 medium supplemented with $10 \mathrm{mM}$ TES-NaOH $(\mathrm{pH}=8)$, and bubbled with a mix (v/v) of $99 \% \mathrm{~N}_{2}$ and $1 \% \mathrm{CO}_{2}$ at a flow rate of $\sim 150 \mathrm{~mL} \mathrm{~min}{ }^{-1}$. Pre-cultures from shake flasks were used for inoculation of cultures with a $60 \mathrm{~mL}$ working volume (area exposed to the light $=0.0028 \mathrm{~m}^{2}$, height of the water column $=10.32 \mathrm{~cm}$ ) in the multi-cultivator, at an initial $\mathrm{OD}_{730}$ of $\sim 0.05$. Fixed light intensity of $30 \mu \mathrm{mol}$ photons $\mathrm{m}^{-2} \mathrm{~s}^{-1}$ (calibrated at the back of the vessel filled with BG11 medium) was given after inoculation and was increased to $120 \mu \mathrm{mol}$ photons $\mathrm{m}^{-2} \mathrm{~s}^{-1}$ at $\mathrm{OD}_{730} \geq 0.5$. Samples were collected daily for both external $\mathrm{OD}_{730}$ determination and for extracellular broth analysis.

\section{Photonfluxostat}

Photonfluxostat is a method for light-limited cyanobacterial batch cultivation at different, yet constant, growth rates [41]. It is based on the dosage of light proportionally to the cell density such that the emitted light per unit of biomass is constant. Performed in a Multi-Cultivator, with its built-in OD sensor, the photonfluxostat illumination regime is implemented by an open-source in-house software package (https://gitlab.com/mmp-uva/pycul tivator), which supervises online $\mathrm{OD}_{720}$ measurements at regular intervals. Operation conditions adopted for the photonfluxostat were similar to the ones described above for the batch cultivations except for the light intensity settings. Briefly, light intensity was also initially set to $30 \mu \mathrm{mol}$ photons $\mathrm{m}^{-2} \mathrm{~s}^{-1}$ immediately after inoculation. But when $\mathrm{OD}_{720}$ reached 0.6, the light intensity was automatically adjusted every $5 \mathrm{~min}$ to ensure that the light intensity per $\mathrm{OD}_{720}$ was constant. This light regime was then maintained until the maximum capacity of the LED panel was reached $(\sim 300 \mu$ mol photons $\mathrm{m}^{-2} \mathrm{~s}^{-1}$, as measured at the back of the vessel filled with BG11 medium). During photonfluxostat cultivation, we grew 12 independent cultures, at different growth rates, via illumination at different light intensities per biomass concentration [specifically, 30 (twice), 40, 45 (twice), 50, 60 (twice), 70, 80, 90, $100 \mu \mathrm{mol}$ photons $\mathrm{m}^{-2} \mathrm{~s}^{-1} \mathrm{OD}^{-1}$ ]. Supernatant samples were collected at regular intervals for analysis of the acetate concentration.

\section{Turbidostat cultivation}

We studied the genetic stability of our strains in populations maintained under turbidostat mode [42]. In this continuous cultivation method, microbial populations are kept at a fixed biomass density by diluting the culture with fresh medium at the same rate as the populations grows. This feedback loop applies a strong selection pressure on cells to grow at the maximal specific growth rate [43]. The turbidostat setup used in this experiment is based on a modified Multi-Cultivator, with additional pumps (Reglo ICC, ISMATEC, Germany) transferring fresh medium to the cultures and, subsequently, to a waste container (i.e., as in a classical chemostat). The "pycultivator" software package that controls the Multi-Cultivator and adjunct hardware additionally sets the pumps to dilute the cultures if the selected $\mathrm{OD}_{720}$ threshold is reached. Cells from pre-cultures in shake flasks were inoculated at $\mathrm{OD}_{720} \sim 0.05$ in four independent cylindrical vessels of the Multi-Cultivator, using the same conditions as specified before, except for the incident light intensity, which was fixed at $50 \mu \mathrm{mol}$ photons $\mathrm{m}^{-2} \mathrm{~s}^{-1}$. The $\mathrm{OD}_{720}$ was recorded every $5 \mathrm{~min}$. When the threshold of $\mathrm{OD}_{720}>0.35$ was reached, cultures were diluted by $8 \%(\mathrm{v} / \mathrm{v})$ with fresh BG11. Strain stability was assessed by monitoring the growth rate and acetate production in time. The growth rate was calculated by fitting a linear function through the natural logarithm of the $\mathrm{OD}_{720}$ during each cell "growth-dilution" cycle. Samples for acetate production were collected periodically throughout the cultivation period. The variation in growth rate, expressed in percentage, was calculated relative to the one observed at the beginning of the cultivation experiment. A similar approach was used to analyze the variability in acetate productivity.

\section{Assay of acetate concentration in spent medium}

Extracellular acetate concentration was determined in samples collected from cultures of the different strains under different conditions using a commercial enzymatic acetate assay (Megazyme). Cells were removed 
from $0.5 \mathrm{~mL}$ culture sample by centrifugation for $5 \mathrm{~min}$ at $13,000 \mathrm{rpm}$ at $4{ }^{\circ} \mathrm{C}$. Supernatant samples were stored at $-20{ }^{\circ} \mathrm{C}$ for later use. For the enzymatic acetate assay, approximately $200 \mu \mathrm{L}$ of a supernatant was used according to the manufacturer's instructions. During the assay, the conversion of the acetate present in the sample to acetyl phosphate is stoichiometrically coupled to the conversion of $\mathrm{NADH}$ to $\mathrm{NAD}+$. The latter conversion was quantitated via the absorption $(340 \mathrm{~nm}$ ) of the sample, which was performed in 96-well plates (Greiner), using a plate reader (BMG FLUOstar OPTIMA Microplate Reader), operated at room temperature. For quantification, the assay was calibrated with a standard curve obtained under the same conditions.

\section{Theory and in silico analysis}

\section{Obligate coupling of product formation to the flux} through anabolic pathways

Several reactions within anabolic pathways have side products, besides the intermediates that are synthesized to eventually be incorporated in various biomass components. Cells tend to be equipped with metabolic routes to reintroduce these side products back into metabolism, as this generally enables a more efficient use of resources (Fig. 1a). This recycling is particularly important when these side products are related to burdensome cellular processes such as carbon fixation by photoautotrophic organisms. This may also partly explain why organisms like Synechocystis tend to have an extended anabolic versatility underlying their very limited number of auxotrophies [44].

The strategy to achieve growth-coupled production explored in this study relies on the disruption of the endogenous reactions that are responsible for the recycling of the side products, which are stoichiometrically associated with the synthesis of biomass precursors (e.g., amino acids, co-factors, lipids). The growth of such mutant strains obviously still requires the production of the biomass precursors and, consequently, formation of the side products. But since the latter cannot be reintroduced into metabolism (as the enzyme(s) carrying this out were disrupted), these very specific compounds will accumulate (Fig. 1b). This may happen intracellularly and/or in the culture, depending on the presence of a transport system, on the thermodynamic drive, and/or on the tendency of the particular compound to leak across the membrane. Either way, in such strains, growth-coupled production is ensured as the synthesis of a side product is tightly coupled to that of an essential biomass precursor.

\section{Introducing "FRUITS"}

We have developed an algorithm to 'Find Reactions Usable in Tapping Side-products'-FRUITS (Additional file 1: Figure S1). It analyzes existing GSMs and enables the identification of side products of anabolism that are suitable to be coupled to growth of the cells by deletion of their respective re-utilization pathway(s) (Fig. 1b). A GSM of the desired production organism, suitably constrained (e.g., mimicking industrial conditions), is the only required input. Applying the pipeline, implemented as a series of Python scripts, FRUITS produces (i) a list of all the modeled metabolites that can be produced in a growth-coupled manner, (ii) the gene deletions that are required to do so, and (iii) the computed maximal biomass formation rate along with the predicted minimum flux toward the identified target compounds.

FRUITS is initiated by evaluating the input GSM for reactions responsible for the synthesis of macromolecules (e.g., proteins, DNA or RNA) and their precursors (e.g., amino acids or nucleotides). All identified reactions are then dissected into their required substrates and products. A distinction for the products is drawn between biomass precursor metabolites (i.e., strictly used within the anabolic pathway) and co-produced molecules (i.e., that are not necessarily directly used as a substrate in an anabolic pathway). The list of co-produced compounds is then trimmed by removing molecules that do not contain carbon (e.g., $\mathrm{Mg}^{2+}, \mathrm{NH}_{4}{ }^{+}$or $\mathrm{PO}_{4}{ }^{3-}$ ) or that are co-factors that act as energy carriers or redox equivalents (e.g., ATP, NADPH, NADH or CoA). The remaining metabolites constitute the list of preliminary growthcoupled candidates that will be considered in subsequent steps of the pipeline.

To ensure that compound excretion can be predicted using a GSM, it is required that it can be exchanged within the boundaries of the defined system. In modeling terminology, this means that a metabolite must be a boundary species for which a so-called sink reaction is present. The metabolites in the preliminary list of candidates do not necessarily have a sink reaction, which would not allow them to ever be predicted to accumulate. This is resolved by creating a model version for each compound of the list in which a single new sink reaction is added, if necessary, for the respective target compound. These multiple models with the appropriate sink reaction present are then further analyzed individually.

The possibility of changing the topology of the metabolic network of each of these models, such that the respective candidate compound is made in a strict growth-coupled way, is then evaluated. In essence, we want to find whether there are gene deletions that completely disrupt the re-utilization of a target compound, and if so, which one(s). This is achieved using an in-house 
Python implementation of OptKnock [33], extended for gene deletions [45]. The user can choose the maximum number of permitted deletions such that it satisfies experimental limitations. OptKnock is very useful to identify the gene deletions necessary to maximize the flux toward a target compound; however, it does not guarantee that the product is uniquely coupled to an anabolic pathway. The strict stoichiometric coupling between the rate of formation of biomass and product are therefore tested by additionally performing FVA on the constrained model with the proposed gene deletions, while using the maximization of biomass formation as the objective function. If the minimum flux rate through the exchange reaction is greater than zero, then the possibility of making this metabolite growth coupled is confirmed (at least in silico!).

Ultimately, this algorithm will report a list of all the identified target compounds along with the necessary associated gene knockouts and the maximal biomass and minimum target product formation rates. This information can then be used directly to guide the engineering of stable producers, and/or be combined with alternative metabolic engineering strategies (e.g., heterologous expression) that may further boost productivity.

\section{Results and discussion}

\section{Analysis of the GSM of Synechocystis using FRUITS}

We tested the newly developed FRUITS using $i$ NN678-a published genome-scale metabolic model of Synechocystis-with default constraints that support a light-limited growth rate of $0.052 \mathrm{~h}^{-1}[30,35]$. The first step of the pipeline of identifying side products resulted in a list of 80 preliminary candidates (Additional file 1: Table S2) out of a total of 817 metabolites present in the model. Next, while allowing a maximum of four gene deletions, we found nine target metabolites (Table 2) as the output of FRUITS. The predicted product yield on biomass $\left(Y_{\mathrm{p} / \mathrm{x}}\right)$ of each target metabolite was calculated based on its production rate and the maximal growth rate. In this particular case, for all the nine metabolites, FVA showed that the minimum and maximum flux rates predicted were identical, suggesting indeed a very strict stoichiometric coupling-just as intended.

The output of FRUITS, when applied to $i$ NN678, reveals some interesting patterns. For instance, the higher the maximum number of allowed gene deletions, not only the greater is the number of target products that are identified, but also the greater are the predicted production rates (see the case of fumarate in Table 2). Here, we have limited this parameter to four, in pace with the common ability to genetically engineer these strains in the laboratory [46]. Another observed pattern is the inverse proportionality between the predicted maximal growth rates and the production rates normalized over the number of carbon atoms per molecule $(\mathrm{C}-\mathrm{mol})$-i.e., the more the carbon is deviated toward product formation, the slower will the culture grow (Fig. 2). This in silico prediction is consistent with a recent study in which the same effect was observed for lactate production [14]. There, a clear drop in growth rate was observed when the flux toward lactate was allosterically increased without changing the expression level of the responsible enzyme ( $\mathrm{L}$-lactate dehydrogenase).

The list of target compounds identified by FRUITS mostly contains entries for which a strategy to achieve growth-coupled production has not been proposed yet. To the best of our knowledge, the only exception is fumarate, which had been previously singled out [23]. However, it is important to note that FRUITS has here revealed additional gene deletions not considered in the previous report, which are predicted to lead to even higher production rates of fumarate. Many of the

Table 2 Metabolites that can be produced in a growth-coupled way based on in silico simulations

\begin{tabular}{|c|c|c|c|}
\hline Metabolite & Growth rate $\left(\mathrm{h}^{-1}\right)$ & Yield $\left(\mathrm{mmol} \mathrm{gDW}^{-1}\right)$ & Gene knockouts \\
\hline 5-Methylthioadenosine & 0.052 & 0.007 & sll0135 \\
\hline Acetate & 0.052 & 0.195 & sll0542, sll1299 \\
\hline Mercaptopyruvate & 0.034 & 5.702 & sll1027 or sll1502, slr0710, sll1499 \\
\hline 5'-Deoxyadenosine & 0.052 & 0.044 & sll1185 \\
\hline 3,4-Dihydroxy-2-butanone 4-phosphate & 0.051 & 0.732 & sll0753, sll0330, s\|11556 \\
\hline Adenine & 0.052 & 0.032 & sll1430 \\
\hline Adenosine & 0.052 & 0.032 & sll1430 \\
\hline S-Adenosyl-_-homocysteine & 0.052 & 0.025 & sll1758 \\
\hline \multirow[t]{3}{*}{ Fumarate } & 0.051 & 0.848 & slr0018 \\
\hline & 0.044 & 3.162 & slr0018, slr0458, sll1349 \\
\hline & 0.043 & 3.509 & slr0018, slr0458, sll1349, slr1755 \\
\hline
\end{tabular}




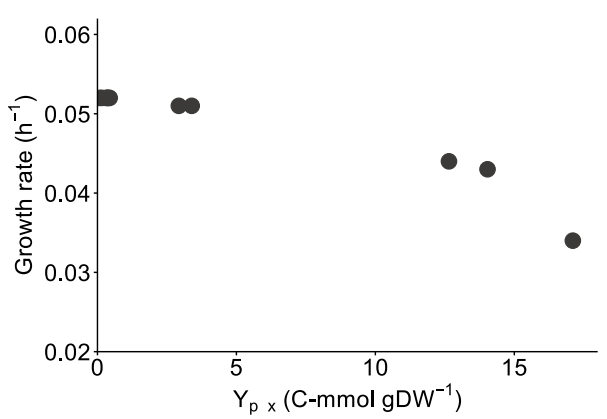

Fig. 2 Relationship between predicted growth rate and carbon product yield on biomass for each of the compounds listed by FRUITS to be coupleable to growth in Synechocystis. Each point represents an individual compound (and a mutant strain with dedicated gene deletions as indicated in Table 2)

compounds in the list are of biotechnological interest, covering a variety of potential applications. For instance, adenosine and adenine can be used for pharmaceutical and nutritional applications [47], respectively, while fumarate and acetate have been listed as relevant for the biobased economy $[48,49]$. The fact that all of them can now in principle be produced directly from $\mathrm{CO}_{2}$ in a sustainable and stable fashion may be of great value.

We experimentally validated the feasibility of this approach for one of the compounds in the list-acetate-by introducing the required genetic modifications and monitoring their effects on biomass and product formation rates. Acetate has a large market size (e.g., 13 million tons in 2015) reflecting its multiple industrial applications, ranging from food additive to chemical intermediate ingredient [48]. In biotechnological processes, it may serve as an accessible carbon source that has been shown to be easily converted into compounds such as poly-3-hydroxybutyrate $[50,51]$ and isopropanol [11]. Additionally, acetate is often adopted as a cross-fed carbon source in co-culture systems $[52,53]$.

\section{Acetate metabolism in Synechocystis}

According to the model $i$ N 678 [30], under light-limited conditions, acetate is produced as a by-product of four independent anabolic pathways (Fig. 3a): (i) the reaction catalyzed by $O$-acetyl-L-homoserine acetate-lyase that converts $\mathrm{O}$-acetyl-L-homoserine to L-homocysteine, an intermediate of methionine synthesis; (ii) in the synthesis of cysteine, through which cysteine and acetate are generated from acetyl serine and hydrogen sulfide, catalyzed by cysteine synthase (encoded by sll0712 or slr1842); (iii) during the step catalyzed by UDP-3-O-acetylglucosamine deacetylase (encoded by sll1508) for lipid A synthesis; and (iv) in the route of biosynthesis of vitamin $B_{12}$, during the step catalyzed by precorrin-6A synthase. The flux through each of these pathways is predicted by FBA to be responsible for $50,23.9,25.9$ and $0.2 \%$, respectively, of the total flux toward acetate. It is important to clarify that under these conditions, none of these predicted fluxes toward
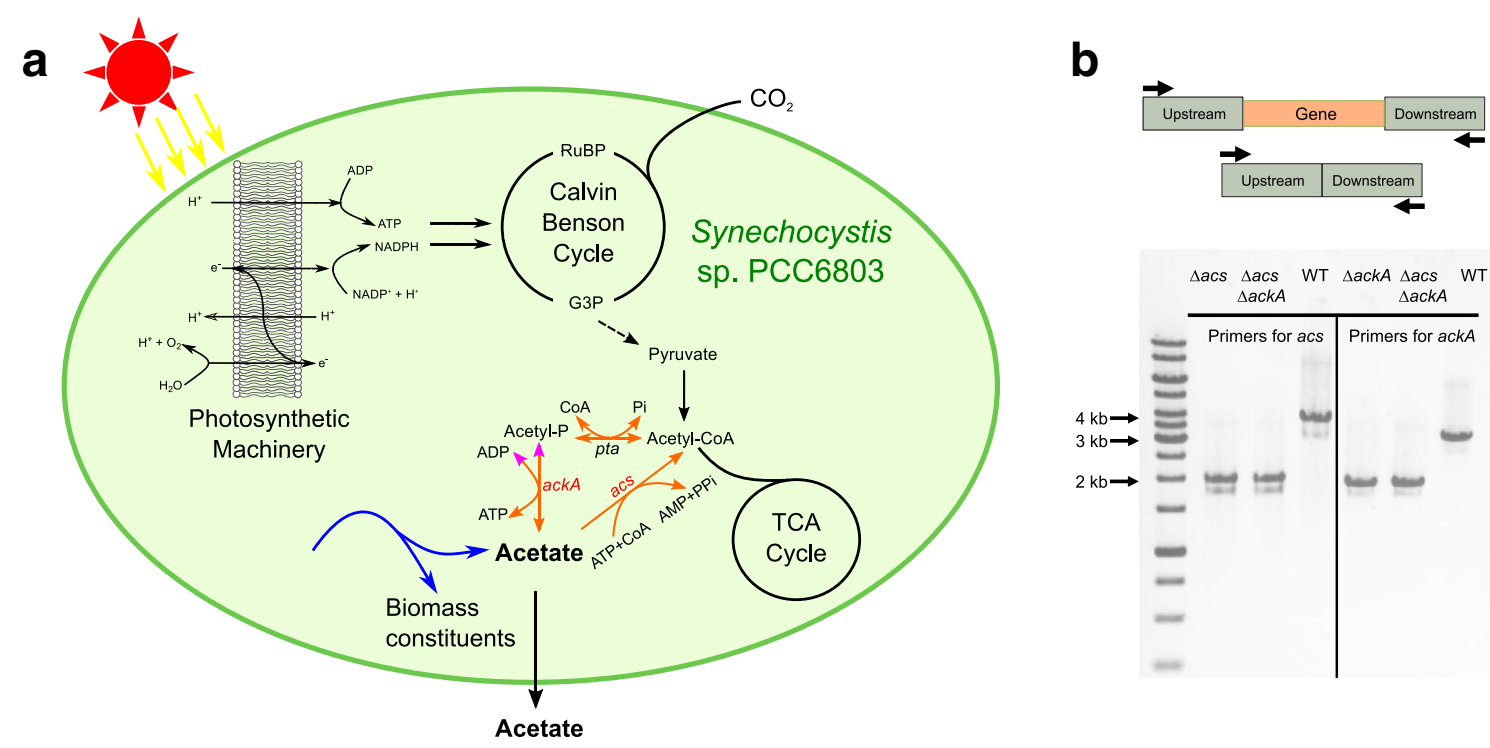

Fig. 3 Acetate metabolism and mutant construction in Synechocystis. a Schematic representation of acetate producing (blue) and consuming (orange) pathways, revealed by the genome-scale model (GSM) [30]. Acetate is also produced as an anabolic by-product and secreted (by an as of yet unknown mechanism). Genes (red and italic) related to acetate consuming pathways were deleted in this study. Pink arrowheads indicate the direction of the AckA predicted in the GSM, but found to be infeasible under the experimental conditions tested. $\mathbf{b}$ PCR confirmation of the strains constructed for markerless deletion of acs and ackA. With the primers on each side of the upstream and downstream homologous region $(\sim 1 \mathrm{~kb}$ each), a markerless construct gave a PCR product $\sim 2 \mathrm{~kb}$, while the wild-type band was about 4 and $3.2 \mathrm{~kb}$ for acs and ackA, respectively 
acetate directly taps from central carbon metabolism or from appending fermentative pathways. This is further supported by experimental data on acetate formation during diel $\mathrm{L} / \mathrm{D}$ regimes (12 $\mathrm{h}$ light/12 $\mathrm{h}$ dark) [54]. There, during the light period, it is clearly shown that acetate is not formed from glycogen via central carbon metabolism.

The flux through all these four anabolic pathways is obviously required for balanced growth. Since light-limited wild-type Synechocystis cultures do not accumulate acetate, this implies that it must be internally re-assimilated. Given the energetic burden that $\mathrm{CO}_{2}$ fixation carries, it is not at all surprising that these cells have evolved metabolic routes to conserve the energy that has been invested in formation of $\mathrm{C}-\mathrm{C}$ bonds. As described in $i \mathrm{NN} 678$ [30], there are two alternative routes to channel acetate back into the metabolic network hub acetyl-CoA (Fig. 3a). One, directly via the activity of acetyl-CoA synthetase (Acs) encoded by sll0542 that irreversibly converts acetate, CoA and ATP into acetyl-CoA, AMP and pyrophosphate (PPi). Another, via a two-step pathway composed of the (erroneously predicted-evidence provided below) reversible reaction catalyzed by the acetate kinase (AckA) encoded by sll1299, plus the reversible activity of a phosphate acetyltransferase (Pta), encoded by slr2132. The overall stoichiometry of the latter pathway differs from the one catalyzed by Acs only in that ADP and Pi are formed rather than AMP and PPi. According to the output of FRUITS (Table 2), if both of these acetate re-utilization routes would be disrupted, then, and only then, would acetate accumulate in the culture in a growth-coupled way. More precisely, a $Y_{\mathrm{p} / \mathrm{x}}$ of $0.19 \mathrm{mM} \mathrm{gDW}^{-1}$ is predicted.

\section{Acetate production in wild-type Synechocystis and derivative strains}

We tested these in silico predictions by constructing, both alone and in combination, markerless deletion mutants of acs (sll0542) and ackA (sll1299), using an established protocol (Fig. 3b) [38]. Then we cultured wild-type Synechocystis and the $\triangle a c s, \triangle a c k A$, and $\triangle a c s \Delta a c k A$ mutants in a controlled batch photocultivation in a Multi-Cultivator under constant illumination for $300 \mathrm{~h}$. During these cultivations, the biomass and acetate concentrations were monitored daily. The growth curves of the four strains were very similar (Fig. 4a), as predicted by the model (Table 3). With respect to acetate accumulation (Fig. 4b), the wild type and the single $\triangle a c k A$ mutant did not produce detectable amounts, while the single $\Delta a c s$ and the double $\triangle a c s \triangle a c k A$ mutants secreted large amounts of acetate reaching a titer of $>2.5 \mathrm{mM}$ after $300 \mathrm{~h}$. The acetate concentrations measured during photoautotrophic growth of the wild-type, $\triangle a c k A$ and $\triangle a c s \Delta a c k A$ strains were as anticipated (Table 3 ); however, for $\Delta a c s$ they were not. In fact, the mutant carrying a single deletion of acs produces the same levels of acetate as the acs and ackA double mutant. This suggests two things: (i) the acetate formed is indeed not a product of the fermentative AckA-Pta pathway under these conditions with continuous illumination; and (ii) AckA does not play a role under these conditions in the re-utilization of acetate, which implies that the activity of Acs alone is responsible for this. This second conclusion is in direct conflict with the model prediction, and so we decided to explore it further both theoretically and experimentally.

\section{Acs serves as the main metabolic acetate assimilation pathway}

We started by analyzing the reaction catalyzed by AckA, which converts acetate and ATP to acetyl-P and ADP, using a biochemical thermodynamics calculator, eQuilibrator [55]. We then estimated $\Delta G$ as a function of the $\log _{10}$ concentration ratios of ADP to ATP and acetyl-P to acetate, rather than the more commonly used products
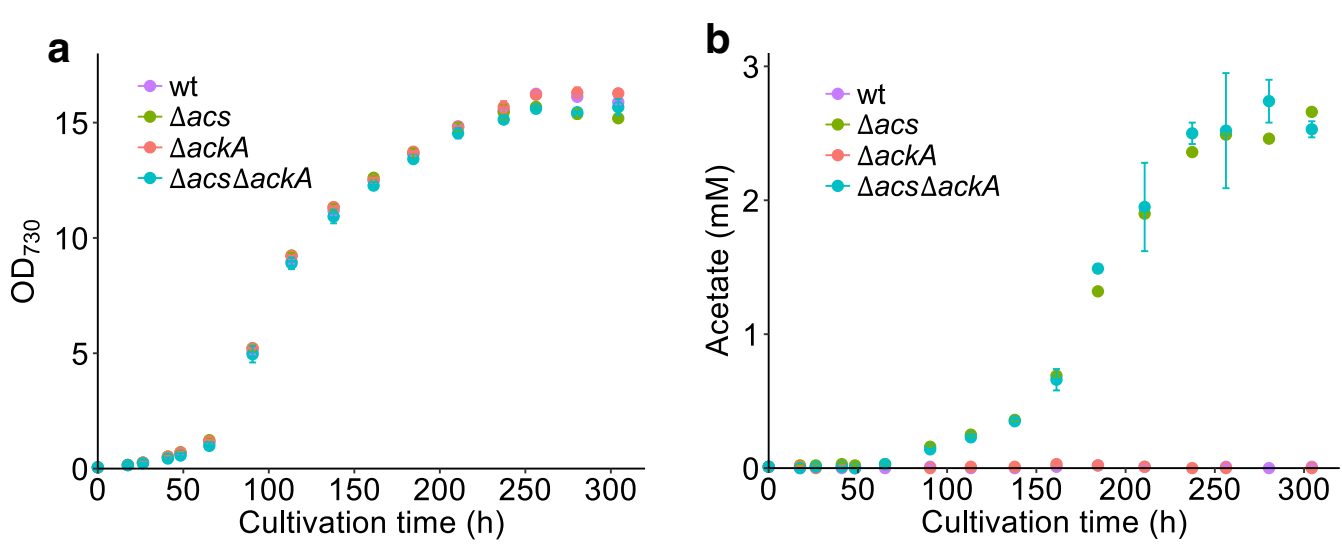

Fig. 4 Acetate production in different Synechocystis strains. Growth curves (a) and acetate concentration (b) in different strains from Multi-Cultivator experiments with continuous illumination. Error bars represent the standard deviation of at least two biological replicates. If an error bar is not visible, it is smaller than the point symbol 
Table 3 Predicted and experimentally observed growth rate and product yield of acetate on biomass in wild-type Synechocystis and derivative strains impaired in acetate metabolism

\begin{tabular}{|c|c|c|c|c|}
\hline \multirow[t]{2}{*}{ Strains } & \multicolumn{2}{|c|}{ Growth rate $\left(\mu, \mathrm{h}^{-1}\right)$} & \multicolumn{2}{|c|}{$\begin{array}{l}\text { Acetate yield }\left(Y_{\mathrm{p} / \mathbf{x}^{\prime}}, \mathrm{mM}\right. \\
\left.\mathrm{gDW}^{-1}\right)\end{array}$} \\
\hline & $\begin{array}{l}\text { Model } \\
\text { prediction }\end{array}$ & Measured & $\begin{array}{l}\text { Model } \\
\text { prediction }\end{array}$ & Measured $^{\mathrm{a}}$ \\
\hline Wild type & 0.052 & $0.048 \pm 0.002$ & 0 & 0 \\
\hline$\triangle a c s$ & 0.052 & $0.049 \pm 0.001$ & 0 & $0.201 \pm 0.011$ \\
\hline$\triangle a c k A$ & 0.052 & $0.048 \pm 0.001$ & 0 & 0 \\
\hline$\triangle a c s a c k A$ & 0.052 & $0.047 \pm 0.002$ & 0.192 & $0.193 \pm 0.007$ \\
\hline
\end{tabular}

a Measured yield was calculated based on data collected during the exponential cell growth phase (65-120 h) presented in Fig. $4 b$. Dry weight concentration was extrapolated from the $\mathrm{OD}_{730}$ measurements using a conversion coefficient determined in this same setup of $148 \mathrm{mg} \mathrm{L}^{-1} \mathrm{OD}_{730}^{-1}[41]$

over substrates, because of the scarcity of data on absolute intracellular metabolite concentrations available for Synechocystis. We calculated that, even considering the highest ATP:ADP ratios reported [56, 57], the concentration of acetate has to be at least $\sim 100$-fold larger than that of acetyl-P for the net flux through the reaction to be positive in the forward direction, i.e., $\Delta G<0$ (Additional file 1: Figure $\mathrm{S} 2$ ). This ratio is highly unlikely under the conditions simulated, and therefore it strongly suggests that the constraints of the AckA reaction (R_ACKr) in the model be revised such that it would be defined as irreversible. When this is done, model predictions and experimental observations are aligned.

We did, however, decide to perform one additional experiment in which pressure would be applied on the thermodynamic drive of AckA in the reverse direction.
This was achieved by supplementing batch cultures of Synechocystis and derivative strains, growing in a MultiCultivator, with an exogenous supply of acetate $(5 \mathrm{mM})$ and monitoring daily the concentrations of both biomass and acetate (Fig. 5a, b). The acetate initially added to the cultures of both wild type and the $\triangle a c k A$ mutant was completely consumed throughout the cultivation, at similar rates for both strains. In contrast, for the $\Delta a c s$ and $\triangle a c s \triangle a c k A$ mutants, the $5 \mathrm{mM}$ acetate initially added appears to not only have remained untouched, but also the extracellular concentration of acetate kept increasing as the cultures became denser.

The evidence provided by these two approaches convinced us that only Acs is required for acetate assimilation. However, the prediction that acetate production in this $\Delta$ acs mutant is coupled to growth still remains to be tested directly.

\section{Acetate production coupled to photoautotrophic growth}

The relationship between the rate of acetate production $\left(q_{\mathrm{p}}, \mathrm{mM}\right.$ acetate $\left.\mathrm{gDW}^{-1} \mathrm{~h}^{-1}\right)$ and the growth rate $(\mu$, $\mathrm{h}^{-1}$ ) for the $\Delta$ acs mutant was explored under constant light-limited conditions during steady-state exponential growth. To achieve this, we applied the new mode of cultivation, the photonfluxostat, by dosing the incident light flux according to the biomass density [41]. This method allows for the light-limited batch photocultivation of photoautotrophs at different, yet constant, growth rates, in which we simultaneously determined the extracellular acetate concentration. We observed a good linear fit $\left(R^{2}=0.902\right)$ for the relationship between $\mu$ and $q_{\mathrm{p}}$ (Fig. 6a). This indicates that the acetate production rate is proportional to the growth rate and, therefore, provides direct evidence that for this strain,
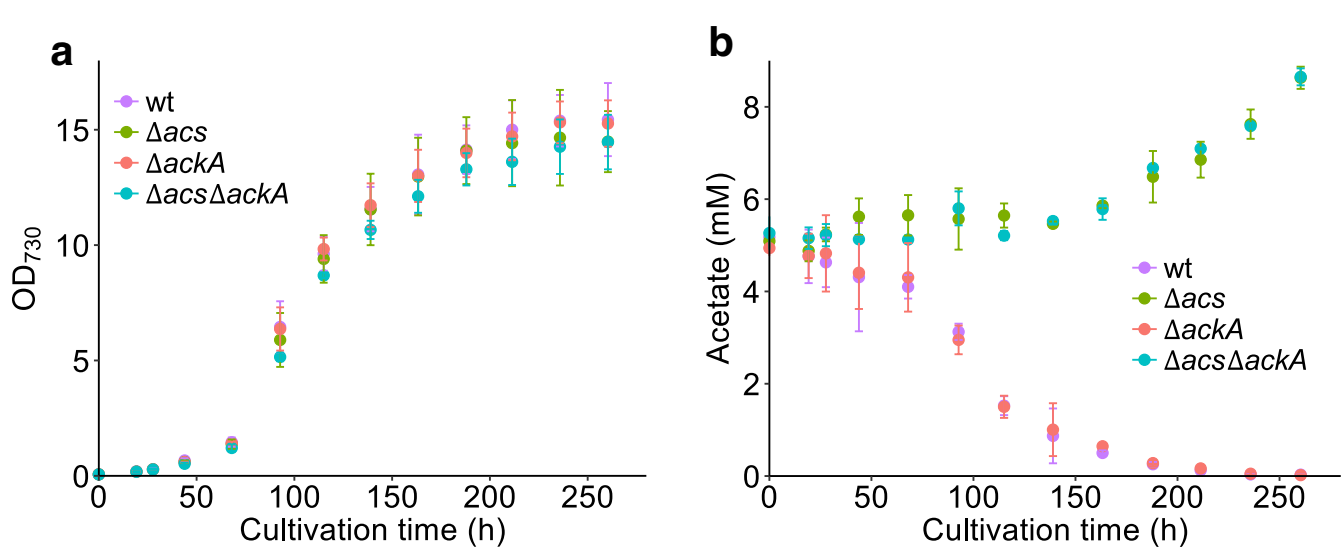

Fig. 5 Acetate consumption/production in wild-type Synechocystis and derivative strains impaired in acetate metabolism while cultured in BG11 medium supplemented with $5 \mathrm{mM}$ acetate. $\mathbf{a}$, b Biomass content and acetate concentration, respectively, in Multi-Cultivator batch experiments under constant illumination. Error bars represent the standard deviation of at least two biological replicates. If an error bar is not visible, it is smaller than the point symbol 
acetate is indeed produced in a strictly growth-coupled fashion.

The metabolic engineering strategy applied here is expected to lead to stable production. This is because the timescale required to evolve an enzyme capable of reutilizing acetate is likely many orders of magnitude larger than the longest biotechnological process one could conceive of. We nonetheless decided to test whether growthcoupled production in fact leads to robust and sustained product formation. For this purpose, four independent biological replicate cultures of the $\Delta a c s$ mutant were tested in prolonged turbidostat experiments, in which there is a strong selection pressure on growth rate [43]. During the cultivation, we monitored online the $\mathrm{OD}_{720}$ from which the growth rate was inferred (Fig. 6b), and periodic samples were taken to determine the rate of acetate production (Fig. 6c). Over the entire course of the cultivation ( $>1$ month), we could not see a statistically significant difference for both the biomass and product formation rates. One could question whether the cultivation time for this experiment was sufficiently long. To answer this it is useful to note how rapidly non-producing mutants can be observed for other production strains in which biomass and product formation rates are not stoichiometrically coupled [14]. Based on this, we argue that if the $\Delta a c s$ mutant would be genetically unstable, in these cultivations there would have been sufficient time to see clear signs of it. We therefore interpret these results as a validation that growth-coupled production
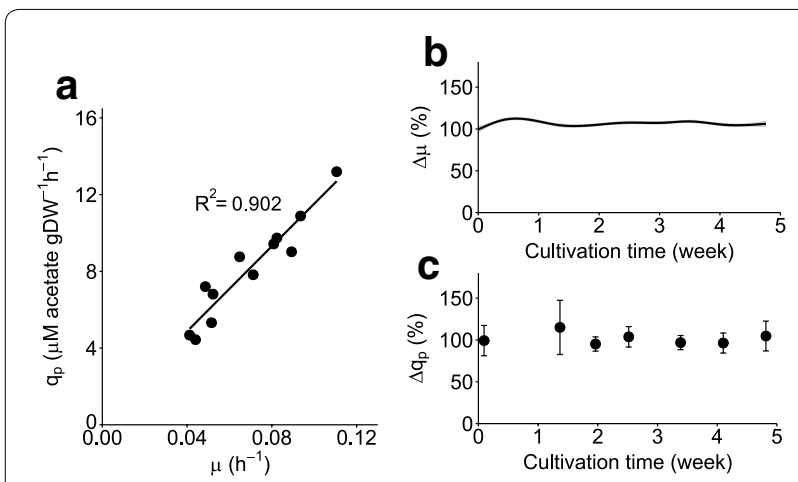

Fig. 6 Stable growth-coupled acetate production in a Synechocystis $\triangle a c s$ mutant. Photonfluxostat cultures at different light intensities per biomass concentration reveal the proportionality between biomass-specific acetate productivity and growth rate (a). Steady states maintained in four independent turbidostat replicates display constant growth rate (b) and biomass-specific acetate productivity (c) for over 1 month of cultivation. Dry weight in a was calculated based on $\mathrm{OD}_{730}$ measurements using a conversion coefficient of $148 \mathrm{mg}$ $\mathrm{L}^{-1} \mathrm{OD}-130$ previously determined for this setup [41]. Each filled circle in $\mathbf{a}$ represents a single observation, while gray shaded area in $\mathbf{b}$ and error bars in $\mathbf{c}$ indicate the standard deviation from four biological replicates indeed has the potential to stabilize the productivity of a target compound, even when the latter is produced at high rates.

\section{Conclusions}

In this study, based on a combination of modeling and experimental approaches, we have been able to engineer the first photoautotrophic growth-coupled cell factory. Its design is based on an algorithm that is general and applicable to any organism for which a GSM is available. The alignment of microbial fitness with product formation is a solution that directly tackles issues of strain instability, which are a major hurdle in the scale-up of many biotechnological processes. The in silico pipeline presented here, along with the proof of principle provided in the form of stable photoautotrophic growth-coupled production, will hopefully elicit wider application of this promising metabolic engineering strategy.

\section{Additional file}

Additional file 1. Additional figures and tables.

\section{Authors' contributions}

FBS first conceived this study along with $\mathrm{KJH}$. WD carried out all strain engineering assisted by DL, and all cultivations supported by JAJ and $\mathrm{HPH}$. CVB was responsible for the implementation of the computational tools and development of the algorithm supervised by BGO and FBS, respectively. WD and FBS wrote the first draft of the manuscript. All authors read and approved the final manuscript.

\section{Author details}

${ }^{1}$ Molecular Microbial Physiology Group, Faculty of Science, Swammerdam Institute for Life Sciences, University of Amsterdam, Science Park 904, 1098 XH Amsterdam, The Netherlands. ${ }^{2}$ Systems Bioinformatics/Amsterdam Institute for Molecules, Medicines and Systems (AIMMS)/Netherlands Institute for Systems Biology, VU University Amsterdam, De Boelelaan 1085, 1081 HV Amsterdam, The Netherlands. ${ }^{3}$ Modelling of Biological Process, BioQuant, Heidelberg University, Im Neuenheimer Feld 267, 69120 Heidelberg, Germany.

\section{Acknowledgements}

The work of Wei Du was supported by the China Scholarship Council. Brett G. Olivier was supported by BE-BASIC Grant F08.005.001. Klaas J. Hellingwerf was supported by the Dutch Ministry of Economic Affairs, Agriculture, and Innovation (research program BioSolarCells). The Netherlands Organization for Scientific Research (NWO) supported Filipe Branco dos Santos through VENI Grant 863.11.019 and, along with Joeri A. Jongbloets, through Solar-2-product grant 733000005

\section{Competing interests}

Klaas J. Hellingwerf is the scientific advisor of Photanol B.V., a University of Amsterdam spin-off company aiming at commercializing sustainable applications with cyanobacteria, in which Hugo Pineda Hernández is now employed as a Junior Researcher. The other co-authors declare that they have no competing interests.

\section{Availability of data and materials}

The strains and datasets used and/or analyzed during the current study are available from the corresponding author on reasonable request.

\section{Ethics approval and consent to participate}

Not applicable. 


\section{Publisher's Note}

Springer Nature remains neutral with regard to jurisdictional claims in published maps and institutional affiliations.

Received: 16 August 2017 Accepted: 31 January 2018

Published online: 13 February 2018

\section{References}

1. Jackson DA, Symons RH, Berg P. Biochemical method for inserting new genetic information into DNA of Simian Virus 40: circular SV40 DNA molecules containing lambda phage genes and the galactose operon of Escherichia coli. Proc Natl Acad Sci USA. 1972;69:2904-9.

2. Curtiss R 3rd. Genetic manipulation of microorganisms: potential benefits and biohazards. Annu Rev Microbiol. 1976;30:507-33.

3. Clomburg JM, Crumbley AM, Gonzalez R. Industrial biomanufacturing: the future of chemical production. Science. 2017;355(6320):aag0804. https://doi.org/10.1126/science.aag0804.

4. Teusink B, Smid EJ. Modelling strategies for the industrial exploitation of lactic acid bacteria. Nat Rev Microbiol. 2006;4:46-56.

5. Darmon E, Leach DR. Bacterial genome instability. Microbiol Mol Biol Rev. 2014;78:1-39.

6. Renda BA, Hammerling MJ, Barrick JE. Engineering reduced evolutionary potential for synthetic biology. Mol BioSyst. 2014;10:1668-78.

7. Angermayr SA, Gorchs Rovira A, Hellingwerf KJ. Metabolic engineering of cyanobacteria for the synthesis of commodity products. Trends Biotechnol. 2015;33:352-61.

8. Cassier-Chauvat C, Veaudor T, Chauvat F. Comparative genomics of DNA recombination and repair in cyanobacteria: biotechnological implications. Front Microbiol. 2016;7:1-13.

9. Ungerer J, Tao L, Davis M, Ghirardi M, Maness P-C, Yu J. Sustained photosynthetic conversion of $\mathrm{CO}_{2}$ to ethylene in recombinant cyanobacterium Synechocystis 6803. Energy Environ Sci. 2012;5:8998.

10. Angermayr SA, Paszota M, Hellingwerf KJ. Engineering a cyanobacterial cell factory for production of lactic acid. Appl Environ Microbiol. 2012:78:7098-106.

11. Kusakabe T, Tatsuke T, Tsuruno K, Hirokawa Y, Atsumi S, Liao JC, et al. Engineering a synthetic pathway in cyanobacteria for isopropanol production directly from carbon dioxide and light. Metab Eng. 2013;20C:101-8.

12. Jacobsen $\mathrm{JH}$, Frigaard NU. Engineering of photosynthetic mannitol biosynthesis from $\mathrm{CO}_{2}$ in a cyanobacterium. Metab Eng. 2014;21:60-70.

13. Jones PR. Genetic instability in cyanobacteria-an elephant in the room? Front Bioeng Biotechnol. 2014;2:12.

14. Du W, Angermayr SA, Jongbloets JA, Molenaar D, Bachmann H, Hellingwerf KJ, et al. Nonhierarchical flux regulation exposes the fitness burden associated with lactate production in Synechocystis sp. PCC6803. ACS Synth Biol. 2016;6:395-401.

15. Feist AM, Zielinski DC, Orth JD, Schellenberger J, Herrgard MJ, Palsson BO. Model-driven evaluation of the production potential for growth-coupled products of Escherichia coli. Metab Eng. 2010;12:173-86.

16. Ou J, Ma C, Xu N, Du Y, Liu X (Margaret). High butanol production by regulating carbon, redox and energy in Clostridia. Front Chem Sci Eng 2015:9:317-23.

17. Fong SS, Burgard AP, Herring CD, Knight EM, Blattner FR, Maranas CD, et al. In silico design and adaptive evolution of Escherichia coli for production of lactic acid. Biotechnol Bioeng. 2005;91:643-8.

18. Shen CR, Lan El, Dekishima Y, Baez A, Cho KM, Liao JC. Driving forces enable high-titer anaerobic 1-butanol synthesis in Escherichia coli. Appl Environ Microbiol. 2011;77:2905-15

19. Jantama K, Haupt MJ, Svoronos SA, Zhang X, Moore JC, Shanmugam $\mathrm{KT}$, et al. Combining metabolic engineering and metabolic evolution to develop nonrecombinant strains of Escherichia coli C that produce succinate and malate. Biotechnol Bioeng. 2008;99:1140-53.

20. Smith KM, Liao JC. An evolutionary strategy for isobutanol production strain development in Escherichia coli. Metab Eng. 2011;13:674-81.

21. Klamt S, Mahadevan R. On the feasibility of growth-coupled product synthesis in microbial strains. Metab Eng. 2015;30:166-78.

22. Gudmundsson S, Nogales J. Cyanobacteria as photosynthetic biocatalysts: a systems biology perspective. Mol Biosyst. 2015;11:60-70.
23. Nogales J, Gudmundsson S, Thiele I. Toward systems metabolic engineering in cyanobacteria: opportunities and bottlenecks. Bioengineered. 2013:4:158-63.

24. Knoop H, Steuer R. A computational analysis of stoichiometric constraints and trade-offs in cyanobacterial biofuel production. Front Bioeng Biotechnol. 2015;3:47.

25. Erdrich P, Knoop H, Steuer R, Klamt S. Cyanobacterial biofuels: new insights and strain design strategies revealed by computational modeling. Microb Cell Fact. 2014;13:128.

26. Shabestary K, Hudson EP. Computational metabolic engineering strategies for growth-coupled biofuel production by Synechocystis. Metab Eng Commun. 2016:3:216-26.

27. Ungerer J, Pakrasi HB. Cpf1 Is a versatile tool for CRISPR genome editing across diverse species of cyanobacteria. Sci Rep. 2016;6:39681.

28. Yao L, Cengic I, Anfelt J, Hudson EP. Multiple gene repression in cyanobacteria using CRISPRi. ACS Synth Biol. 2016;5:207-12.

29. Allahverdiyeva Y, Mustila H, Ermakova M, Bersanini L, Richaud P, Ajlani G, et al. Flavodiiron proteins Flv1 and Flv3 enable cyanobacterial growth and photosynthesis under fluctuating light. Proc Natl Acad Sci USA. 2013;110:4111-6.

30. Nogales J, Gudmundsson S, Knight EM, Palsson BO, Thiele I. Detailing the optimality of photosynthesis in cyanobacteria through systems biology analysis. Proc Natl Acad Sci. 2012;109:2678-83.

31. Orth JD, Thiele I, Palsson B $\varnothing$. What is flux balance analysis? Nat Biotechnol. $2010 \cdot 28 \cdot 245-8$

32. Mahadevan $\mathrm{R}$, Schilling $\mathrm{CH}$. The effects of alternate optimal solutions in constraint-based genome-scale metabolic models. Metab Eng. 2003;5:264-76

33. Burgard AP, Pharkya P, Maranas CD. OptKnock: a bilevel programming framework for identifying gene knockout strategies for microbial strain optimization. Biotechnol Bioeng. 2003:84:647-57.

34. Olivier BG, Rohwer JM, Hofmeyr JH. Modelling cellular systems with PySCeS. Bioinformatics. 2005:21:560-1.

35. Maarleveld TR, Boele J, Bruggeman FJ, Teusink B. A data integration and visualization resource for the metabolic network of Synechocystis sp. PCC 6803. Plant Physiol. 2014;164:1111-21.

36. Boele J, Olivier BG, Teusink B. FAME, the flux analysis and modeling environment. BMC Syst Biol. 2012;6:8.

37. Rippka R, Deruelles J, Waterbury JB, Herdman M, Stanier RY. Generic assignments, strain histories and properties of pure cultures of cyanobacteria. J Gen Microbiol. 1979;111:1-61.

38. Cheah YE, Albers SC, Peebles CAM. A novel counter-selection method for markerless genetic modification in Synechocystis sp. PCC 6803. Biotechnol Prog. 2013;29:23-30.

39. Zhang XZ, Yan X, Cui ZL, Hong Q, Li SP. mazF, a novel counter-selectable marker for unmarked chromosomal manipulation in Bacillus subtilis. Nucleic Acids Res. 2006:34:e71.

40. Zhu T, Xie XM, Li ZM, Tan XM, Lu XF. Enhancing photosynthetic production of ethylene in genetically engineered Synechocystis sp. PCC 6803. Green Chem. 2015;17:421-34

41. Du W, Jongbloets JA, Pineda Hernández H, Bruggeman FJ, Hellingwerf KJ, Branco dos Santos F. Photonfluxostat: a method for light-limited batch cultivation of cyanobacteria at different, yet constant, growth rates. Algal Res. 2016;20:118-25.

42. Bryson V, Szybalski W. Microbial selection. Science. 1952;116:45-51.

43. Bachmann H, Molenaar D, Branco Dos Santos F, Teusink B. Experimental evolution and the adjustment of metabolic strategies in lactic acid bacteria. FEMS Microbiol Rev. 2017:41:S201-19.

44. Branco dos Santos F, Du W, Hellingwerf KJ. Synechocystis: not just a plugbug for $\mathrm{CO}_{2}$, but a green E. coli. Front Bioeng Biotechnol. 2014;2:36.

45. Kim J, Reed JL. OptORF: optimal metabolic and regulatory perturbations for metabolic engineering of microbial strains. BMC Syst Biol. 2010;4:53.

46. Chen X, Schreiber K, Appel J, Makowka A, Fähnrich B, Roettger M, et al. The Entner-Doudoroff pathway is an overlooked glycolytic route in cyanobacteria and plants. Proc Natl Acad Sci. 2016;113:5441-6.

47. Bolton EE, Wang Y, Thiessen PA, Bryant SH. PubChem: integrated platform of small molecules and biological activities. Annu Rep Comput Chem. 2008;4:217-41.

48. Pal P, Nayak J. Acetic acid production and purification: critical review towards process intensification. Sep Purif Rev. 2017:46:44-61. 
49. Werpy T, Petersen G. Top value added chemicals from biomass volume I-results of screening for potential candidates from sugars and synthesis gas. US Department of Energy. 2004. https://doi.org/10.2172/15008859.

50. Tyo KE, Jin YS, Espinoza FA, Stephanopoulos G. Identification of gene disruptions for increased poly-3-hydroxybutyrate accumulation in Synechocystis PCC 6803. Biotechnol Prog. 2009;25:1236-43.

51. Thiel K, Vuorio E, Aro E-M, Kallio PT. The effect of enhanced acetate influx on Synechocystis sp. PCC 6803 metabolism. Microb Cell Fact. 2017;16:21.

52. Zhou K, Qiao KJ, Edgar S, Stephanopoulos G. Distributing a metabolic pathway among a microbial consortium enhances production of natural products. Nat Biotechnol. 2015;33:377.

53. Therien JB, Zadvornyy OA, Posewitz MC, Bryant DA, Peters JW. Growth of Chlamydomonas reinhardtii in acetate-free medium when co-cultured with alginate-encapsulated, acetate-producing strains of Synechococcus sp. PCC 7002. Biotechnol Biofuels. 2014;7:154.
54. Angermayr $S A$, van Alphen $P$, Hasdemir $D$, Kramer $G$, lqbal M, van Grondelle W, et al. Culturing Synechocystis sp. strain PCC 6803 with N and $\mathrm{CO}_{2}$ in a diel regime reveals multiphase glycogen dynamics with low maintenance costs. Appl Environ Microbiol. 2016;82:4180-9.

55. Flamholz A, Noor E, Bar-Even A, Milo R. eQuilibrator-the biochemical thermodynamics calculator. Nucleic Acids Res. 2012;40(D1):D770-5.

56. Kallas T, Castenholz RW. Internal pH and ATP-ADP pools in the cyanobacterium Synechococcus sp. during exposure to growth-inhibiting low pH. J Bacteriol. 1982;149:229-36.

57. Merida A, Leurentop L, Candau P, Florencio FJ. Purification and properties of glutamine synthetases from the cyanobacteria Synechocystis sp. strain PCC 6803 and Calothrix sp. strain PCC 7601. J Bacteriol. 1990;172:4732-5.

58. Carpine R, Du W, Olivieri G, Pollio A, Hellingwerf KJ, Marzocchella A, et al. Genetic engineering of Synechocystis sp. PCC6803 for poly- $\beta$ hydroxybutyrate overproduction. Algal Res. 2017;25:117-27.

\section{Submit your next manuscript to BioMed Central and we will help you at every step:}

- We accept pre-submission inquiries

- Our selector tool helps you to find the most relevant journal

- We provide round the clock customer support

- Convenient online submission

- Thorough peer review

- Inclusion in PubMed and all major indexing services

- Maximum visibility for your research

Submit your manuscript at www.biomedcentral.com/submit 